\title{
The Study of the Static Stability of Complex Electrical Systems
}

\author{
Kaxramon R. Allaev and Tokhir F. Makhmudov* \\ Tashkent State Technical University named after Islam Karimov \\ 2 University Str., Tashkent, 100095, Uzbekistan
}

Received 10.03.2018, received in revised form 01.07.2019, accepted 14.11.2019

The article shows the effectiveness of the joint application of the equations of node voltages (ENV) and the Lyapunov's function in a quadratic form for the analysis of small oscillations of a power system, in the literature called method Allaev. The joint solution of the equations of node voltages and Lyapunov's matrix equation allows to determine the stability conditions of the electrical system and identify the generator, which first approaches the stability limit. As will be shown below, studies of small oscillations of complex electrical systems can be carried out in full on the basis of matrix methods, successfully developed in recent decades. This is facilitated by the removal of memory limitations and a sharp increase in the speed of computing modern computers.

Keywords: static stability, electric system, equations of node voltages, small oscillations, Lyapunov's function in quadratic form, Sylvester's theorem.

Citation: Allaev K.R., Makhmudov T.F. The study of the static stability of complex electrical systems, J. Sib. Fed. Univ. Eng. technol., 2019, 12(8), 929-938. DOI: 10.17516/1999-494X-0192.

\section{Исследование статической устойчивости \\ сложных электрических систем}

\author{
К.Р. Аллаев, Т.Ф. Махмудов \\ Ташкентский государственный технический \\ университет имени Ислама Каримова \\ Узбекистан, 100095, Ташкент, ул. Университетская, 2
}

В статье показана эффективность совместного применения уравнений узловых напряжений (УУН) и функиии Ляпунова в квадратичной форме для анализа мальх колебаний электрической системы, в литературе получившего название метода Аллаева. Совместное решение уравнений узловых напряжений и матричного уравнения Ляпунова позволяет определить условия

(C) Siberian Federal University. All rights reserved

This work is licensed under a Creative Commons Attribution-NonCommercial 4.0 International License (CC BY-NC 4.0).

* Corresponding author E-mail address: tox-05@yandex.com 
устойчивости электрической системы и выявить генератор, который первым приближается к пределу устойчивости. Исследования малых колебаний сложных электрических систем могут быть выполнены в полном объеме на основе матричных методов, успешно разработанных в последние десятилетия. Этому способствует устранение ограничений памяти и резкое увеличение скорости вычисления современных компьютеров.

Ключевые слова: статическая устойчивость, электрическая система, уравнения узловых напряжений, малье колебания, функиия Ляпунова в квадратичной форме, теорема Сильвестра.

\section{Введение}

Рост производства в промышленности и сфере услуг требует, как правило, увеличения потребления электроэнергии, что, в свою очередь, зависит от ввода в эксплуатацию новых генерирующих мощностей, линий электропередач и оборудования подстанций. Новое оборудование, установленное на предприятиях, и новые бытовые потребители предполагают использование более качественной электроэнергии по сравнению с той, что поставляется потребителям в ряде районов в настоящее время.

Наивысшую опасность, нарушающую нормальное электроснабжение, представляет аварийный режим в электроэнергетической системе (ЭЭС), называемый системной аварией [1]. При такой аварии прекращает работу все оборудование, использующее электроэнергию, что приводит не только к недовыработке, но и к огромным убыткам от брака и порчи продукции, а также к аварии самого производящего оборудования.

Следует заметить, что системные аварии могут произойти незаметно, без видимых причин, при нормальной работе электрической системы. Такие аварии связаны с подбором неправильных коэффициентов регулирующих устройств, наличием устройств продольной компенсации и т.д., с сущностью энергетических систем, являющихся сложными техническими системами.

По энергетической иерархии должно осуществляться правильное электроснабжение: генерация, транспортирование и распределение электроэнергии, чтобы обеспечить ее должное качество при потреблении.

Для решения этих задач необходимо постоянно оценивать статическую устойчивость ЭЭС или ее устойчивость к «малым» колебаниям, так как именно нарушения такой устойчивости приводят к системным авариям с их отрицательными последствиями [2].

Проблема исследования статической устойчивости современных сложных электрических систем усложняется в связи с наличием в их составах слабых связей, разнообразных регулирующих устройств, препятствующих определению общей настройки, агрегатов с различными постоянными времени и т.д. [3, 4]. В настоящее время для решения практических задач применяют методы, основанные на вычислении синхронизирующей мощности одной из электростанций системы, т.е. определяющей апериодическую устойчивость в предположении отсутствия самораскачивания [5].

Применим матричный метод, основанный на функциях Ляпунова в квадратичной форме и УУН (метод Аллаева), для решения задачи статической устойчивости сложных электрических систем [3]. 
Широкое внедрение мощных и быстрых цифровых компьютеров в практику диспетчерских и исследовательских расчетов и особенно перспективы их развития [3] устраняют ограничения на использование более трудоемких вычислительно эффективных, но строгих методов анализа устойчивости. Эти обстоятельства создали хорошие предпосылки для применения метода функций Ляпунова в квадратичной форме для анализа малых колебаний сложных ЭЭС.

Расчетный анализ статической устойчивости ЭЭС различной сложности показывает, что наиболее строгим теоретически, удобным для вычислений и эффективным по результатам является использование двух фундаментальных методов - метода функций Ляпунова в квадратичной форме и узловых уравнений $[3,6]$. Тем более методы изучения малых колебаний с учетом самораскачивания сложны, поэтому достаточно надежные результаты могут быть получены при строгом математическом описании системы управления для контролируемых объектов с использованием их параметров и характеристик [7, 8].

\section{Методы исследования}

Обзор публикаций [3, 4, 6, 9-11] показывает, что применение матричных уравнений Ляпунова и Риккати расширяется, так как роль матричных методов исследования линейных динамических систем возрастает, что связано с разработкой алгоритмов и программ, численных и аналитических методов для их решения. Особое внимание следует уделить интенсивным исследованиям, приводящим к новым и чрезвычайно эффективным методам решения матричных уравнений (в том числе аналитических, основанных на технологии вложения систем - канонизации матриц), широко используемым на практике [9, 11].

Известно $[4,6,9]$, что функция Ляпунова в квадратичной форме для линейных дифференциальных уравнений единственная, которая обеспечивает необходимые и достаточные условия устойчивости исследуемой системы при возникновении в ней малых возмущений. Поэтому основой исследований в этой работе является функция Ляпунова в квадратичной форме и узловые уравнения (метод Аллаева), а предметом исследования служат линеаризованные дифференциальные уравнения элементов ЭЭС [3]. Матричные уравнения элементов электрических систем, являющихся основной частью ЭЭС, составлены на основе уравнений переменных состояния, которые получили наибольшее распространение. Рассматриваемые матричные уравнения используются для анализа переходных процессов и статической устойчивости ЭЭС и синтеза оптимальных параметров регуляторов синхронных машин, работающих в сложной электрической системе.

Установившийся режим исследуемой ЭЭС определяется на основе уравнений узловых напряжений. Узловые уравнения, имеющие функциональную связь между токами и напряжениями узлов, наиболее полно описывают электрическое состояние сети любой сложности [10].

В общем случае узловые уравнения можно записать в виде [7, 10]

$$
\mathrm{YU}=\mathrm{I}+\mathrm{Y}_{\mathrm{i} 0} \mathrm{U}_{0}+\mathrm{J}^{*}
$$

где

$$
\mathrm{Y}=\left[\begin{array}{rrrr}
\mathrm{y}_{11} & -\mathrm{y}_{12} & \ldots & -\mathrm{y}_{1 \mathrm{n}} \\
-\mathrm{y}_{12} & \mathrm{y}_{22} & \ldots & -\mathrm{y}_{2 \mathrm{n}} \\
-\mathrm{y}_{\mathrm{n} 1} & -\mathrm{y}_{\mathrm{n} 2} & \ldots & \mathrm{y}_{\mathrm{nn}}
\end{array}\right]
$$


матрица проводимости исследовательской системы; I, Y ственно, узловых токов, проводимостей связи с балансирующим узлом, источников тока, представляющих собой поперечные ветви с заданными проводимостями.

Для решения узловых уравнений выберем метод Ньютона в полярных координатах, к преимуществам которого можно отнести квадратичную сходимость итерационных процессов, возможность дальнейшего использования для решения задач оптимизации и расчета устойчивости [10]. Кроме того, напряжения узлов $U_{\mathrm{j}}$ и углы нагрузки генераторов $\delta_{\mathrm{j}}$, которые используются в уравнениях Ляпунова, определяемые на основе решения узловых уравнений, содержат всю информацию о состоянии системы, какой бы сложной она ни была [11].

На основе вычисленных значений напряжений генераторных узлов и узлов, содержащих вращающиеся машины, последовательно проверяется положительность матриц квадратичных форм Ляпунова, устанавливающих выполнение необходимых и достаточных условий статической устойчивости генераторов (станций) и ЭЭС. По существу, задача анализа статической устойчивости сложной ЭЭС сводится к многократному исследованию схемы «генератор - шины», которая в практических расчетах описывается уравнением порядка не более 4-15.

В классическом случае уравнения, описывающие процессы в ЭЭС, являются однородными линейными (линеаризованными) дифференциальными уравнениями и имеют вид $[5,6]$

$$
\begin{aligned}
\frac{d x_{1}}{d t} & =a_{11} x_{1}+a_{12} x_{2}+\cdots \cdots \cdots+a_{1 n} x_{n} \\
\frac{d x_{2}}{d t} & =a_{21} x_{1}+a_{22} x_{2}+\cdots \cdots \cdots+a_{2 n} x_{n} \\
\frac{d x_{n}}{d t} & =a_{n 1} x_{1}+a_{n 2} x_{2}+\cdots \cdots \cdots \cdots+a_{n n} x_{n}
\end{aligned}
$$

либо в матричной форме

$$
\dot{\mathrm{x}}=\mathrm{Ax},
$$

где

$$
\mathrm{A}=\left|\begin{array}{cc}
\mathrm{a}_{11} & \mathrm{a}_{12} \ldots \ldots \ldots \mathrm{a}_{1 \mathrm{n}} \\
\mathrm{a}_{21} & \mathrm{a}_{22} \ldots \ldots \ldots . \mathrm{a}_{2 \mathrm{n}} \\
\ldots \ldots \ldots \ldots \ldots \ldots \ldots \ldots & \ldots \ldots \ldots \ldots \ldots \\
\mathrm{a}_{\mathrm{n} 1} & \mathrm{a}_{\mathrm{n} 2} \ldots \ldots \ldots . \mathrm{a}_{\mathrm{nn}}
\end{array}\right|
$$

и $\mathrm{x}^{\mathrm{T}}=\left[\mathrm{x}_{1}, \mathrm{x}_{2}, \ldots, \mathrm{x}_{\mathrm{n}}\right]^{\mathrm{T}}$ - транспонированный вектор переменных состояния.

Для определения устойчивости (4) используем метод Ляпунова и зададим функцию в виде положительно определенной квадратичной формы

$$
\mathrm{V}(\mathrm{x})=\mathrm{x}^{\mathrm{T}} \mathrm{Qx},
$$

или $\mathrm{V}=\sum_{\mathrm{i}, \mathrm{j}=1}^{\mathrm{n}} \mathrm{q}_{\mathrm{ij}} \mathrm{x}_{\mathrm{i}} \mathrm{x}_{\mathrm{j}}$.

Производная этой функции 


$$
\begin{aligned}
& \frac{d V}{d t}=\frac{d\left(x^{T} Q x\right)}{d t}=\left(\frac{d x}{d t}\right)^{T} Q x+x^{T} Q\left(\frac{d x}{d t}\right)= \\
& =(A x)^{T} Q x+x^{T} Q A x=x^{T} A^{T} Q x+x^{T} Q A x= \\
& =x^{T}\left(A^{T} Q+Q A\right) x .
\end{aligned}
$$

Потребуем, чтобы функция Ляпунова удовлетворяла требованию

$$
\frac{\mathrm{dV}}{\mathrm{dt}}=-\mathrm{W},
$$

где $\mathrm{W}=\mathrm{x}^{\mathrm{T}} \mathrm{Cx}$ - произвольная положительно определенная симметрическая матрица.

Приравнивая выражения (10) и (11), получим уравнение

$$
\mathrm{A}^{\mathrm{T}} \mathrm{Q}+\mathrm{QA}=-\mathrm{C} .
$$

Уравнение (9), называемое матричным уравнением Ляпунова, обеспечивает условие устойчивости, если одновременно выполняются неравенства $\mathrm{V}>0$ и $\dot{\mathrm{V}}<0$ в некоторой области пространства переменных $\left(\mathrm{x}_{1}, \mathrm{x}_{2}, \ldots, \mathrm{x}_{\mathrm{n}}\right)$, включающей начало координат [6].

Заметим, что обе матрицы $\mathrm{Q}$ и $\mathrm{C}$ симметричны. Действительно, если матрица $\mathrm{Q}$ симметрична, т. е. $\mathrm{Q}^{\mathrm{T}}=\mathrm{Q}$, то

$$
\mathrm{C}^{\mathrm{T}}=-\left(\mathrm{A}^{\mathrm{T}} \mathrm{Q}+\mathrm{QA}\right)^{\mathrm{T}}=-\mathrm{Q}^{\mathrm{T}} \mathrm{A}-\mathrm{A}^{\mathrm{T}} \mathrm{Q}=-\left(\mathrm{A}^{\mathrm{T}} \mathrm{Q}+\mathrm{QA}\right)=\mathrm{C}
$$

и, следовательно, матрица С симметрична.

Функции (10), где Q - положительно определенная симметрическая матрица, удовлетворяющая условиям теоремы Ляпунова, называются квадратичными функциями Ляпунова. Теорема Ляпунова сводит проверку устойчивости исследуемой системы к решению линейного матричного уравнения. Так как матрица Q симметрична, то уравнение Ляпунова эквивалентно системе $\mathrm{n}(\mathrm{n}+1) / 2$ линейных алгебраических уравнений. При большой размерности матрицы А решение такой системы занимает меньше времени, чем вычисление характеристического многочлена матрицы А [6].

Согласно теореме Сильвестра [1, 5], положительность главных диагональных миноров матрицы коэффициентов Q квадратичной формы (3) является необходимым и достаточным условием для устойчивости рассматриваемой системы при малых возмущениях

$$
Q=\left|\begin{array}{lll}
\mathrm{q}_{11} & \mathrm{q}_{12} & \mathrm{q}_{13} \\
\mathrm{q}_{21} & \mathrm{q}_{22} & \mathrm{q}_{23} \\
\mathrm{q}_{31} & \mathrm{q}_{32} & \mathrm{q}_{33}
\end{array}\right|>0
$$

T.e.

$$
\Delta_{Л 1}=\mathrm{q}_{11}>0, \Delta_{Л 2}=\left|\begin{array}{cc}
\mathrm{q}_{11} & \mathrm{q}_{12} \\
\mathrm{q}_{21} & \mathrm{q}_{22}
\end{array}\right|>0, \ldots \Delta_{Л n}=\left|\begin{array}{cccc}
\mathrm{q}_{11} & \mathrm{q}_{12} & \ldots & \mathrm{q}_{1 \mathrm{n}} \\
\mathrm{q}_{21} & \mathrm{q}_{22} & \ldots & \mathrm{q}_{2 \mathrm{n}} \\
\ldots \ldots \ldots \ldots \ldots \ldots . . & \ldots \ldots \ldots \ldots & \ldots \ldots \ldots \\
\mathrm{q}_{\mathrm{n} 1} & \mathrm{q}_{\mathrm{n} 2} & \ldots & \mathrm{q}_{\mathrm{nn}}
\end{array}\right|>0
$$

Анализ нарушения условия $\Delta_{л 1}=q_{11}>0$ показывает все виды нарушения устойчивости электрической системы (апериодическое нарушение, самовозбуждение, самораскачивание) [6].

$$
-933-
$$


Линеаризованные уравнения простейшей ЭЭС при наличии на синхронном генераторе автоматических регуляторов возбуждения (АРВ) пропорционального или сильного воздействия имеют вид $[4,6]$ :

- уравнение относительного движения ротора синхронной машины:

$$
\mathrm{T}_{\mathrm{j}}\left(\mathrm{d}^{2} \Delta \delta / \mathrm{dt}\right)=-\mathrm{P}_{\mathrm{d}}(\mathrm{d} \Delta \delta / \mathrm{dt})-\Delta \mathrm{P}
$$

- уравнение переходных процессов в обмотке возбуждения:

$$
\mathrm{T}_{\mathrm{d} 0}\left(\Delta \mathrm{E}_{\mathrm{q}}^{\prime} / \mathrm{dt}\right)=\Delta \mathrm{E}_{\mathrm{qe}}-\Delta \mathrm{E}_{\mathrm{q}} ;
$$

- уравнение в обмотке возбуждения возбудителя:

$$
\mathrm{T}_{\mathrm{e}}\left(\Delta \mathrm{E}_{\mathrm{qe}} / \mathrm{dt}\right)=\mathrm{k}_{\mathrm{e}} \Delta \mathrm{e}-\Delta \mathrm{E}_{\mathrm{qe}}
$$

- уравнение усилительного элемента:

$$
\mathrm{T}_{\mathrm{y}}(\Delta \mathrm{e} / \mathrm{dt})=\mathrm{k}_{\mathrm{y}} \Delta \mathrm{u}-\Delta \mathrm{e}
$$

- уравнение измерительного элемента:

$$
\mathrm{T}_{\mathrm{U}}(\mathrm{d} \Delta \mathrm{u} / \mathrm{dt})=\mathrm{k}_{\mathrm{u}} \Delta \mathrm{u}_{\mathrm{r}}-\Delta \mathrm{u}
$$

- уравнение, отражающее влияние АРВ:

$$
\Delta \mathrm{e}=\sum_{j}\left(\mathrm{k}_{0 \Pi \mathrm{j}} \Delta \Pi_{\mathrm{j}}+\mathrm{k}_{1 \Pi \mathrm{j}}\left(\mathrm{d} \Delta \Pi_{\mathrm{j}} / \mathrm{dt}\right)+\mathrm{k}_{2 \Pi \mathrm{j}}\left(\mathrm{d}^{2} \Delta \Pi_{\mathrm{j}} / \mathrm{dt}^{2}\right) ;\right.
$$

здесь $-\mathrm{T}_{\mathrm{j}}, \mathrm{T}_{\mathrm{d} 0}, \mathrm{~T}_{\mathrm{e}}, \mathrm{T}_{\mathrm{y}}, \mathrm{T}_{\mathrm{h}}$ - постоянные инерции агрегата, постоянные времени, соответственно - обмотки возбуждения при разомкнутой обмотке статора, возбудителя, усилительного элемента, преобразовательного и измерительного элементов $\left(\mathrm{T}_{\mathrm{и}}=\mathrm{T}_{\Pi}\right) ; \Delta \delta, \Delta \mathrm{E}_{\mathrm{q}}^{\prime}, \Delta \mathrm{E}_{\mathrm{q}}, \Delta \mathrm{E}_{\mathrm{qe}}, \Delta \mathrm{e}, \Delta \mathrm{u}$, $\Delta \mathrm{u}_{\mathrm{r}}-$ отклонения угла нагрузки, переходной э.д.с., э.д.с. холостого хода, э.д.с. на кольцах ротоpa, напряжения на обкладках возбудителя и напряжения на шинах генератора; $\Delta \prod_{j}-$ параметры режима, по которым осуществляется регулирование возбуждения генератора; $\mathrm{P}_{\mathrm{d}}-$ демпферный коэффициент; $\mathrm{k}_{0 \Pi \mathrm{j}}, \mathrm{k}_{1 \Pi \mathrm{j}}, \mathrm{k}_{2 п \mathrm{j}}$ - коэффициенты усиления по каналам регулирования APB, соответственно - по отклонению, по первой и второй производным параметров режима. Отклонения регулируемого параметра режима генератора или системы определяют по соотношению

$$
\Delta \Pi_{\mathrm{j}}=\left(\mathrm{d} \prod_{\mathrm{j}} / \mathrm{d} \delta\right) \Delta \delta+\left(\Delta \prod_{\mathrm{j}} / \mathrm{dE}_{\mathrm{q}}\right) \Delta \mathrm{E}_{\mathrm{q}} .
$$

\section{Расчетная часть}

Рассмотрим совместное применение уравнений узлового напряжения и функции Ляпунова в квадратичной форме на примере трехузловой схемы (рис. 1). В качестве балансирующего выберем нулевой узел, первый и третий узлы являются генерирующими, второй узел - нагрузочный.

Анализ статической устойчивости сложной ЭЭС проведем на основе известных допущений:

- при вычислении синхронизирующей мощности какого-либо из генераторов углы роторов всех остальных генераторов остаются неизменными. При этом мощности всех генераторов системы изменяются; 


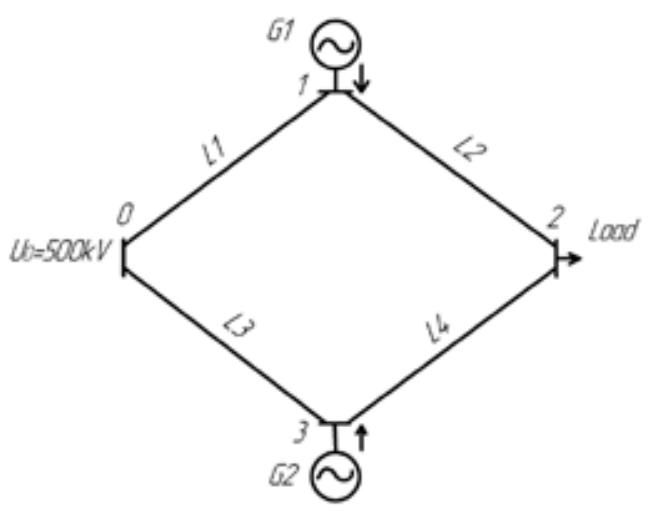

Рис. 1. Принципиальная схема трехузловой электрической системы

Fig. 1. Schematic diagram of a three-node electrical system

- предположим э.д.с. генераторов постоянными для заданного режима, параметры схемы замещения электрической системы и нагрузок постоянны, при этом активные составляющие комплексных сопротивлений не учитываются $(\mathrm{r}=0)$;

- в установившемся режиме работы сложной системы мощности машин могут быть выражены через собственные и взаимные проводимости ветвей схемы замещения электрической системы, которые также считаются постоянными [3].

Как известно, для исследования статической устойчивости сложных систем пользуются позиционной математической моделью ЭЭС, имеющей вид:

- система дифференциальных уравнений относительного движения роторов синхронных генераторов:

$$
\begin{aligned}
& \mathrm{T}_{\mathrm{j} 1} \frac{\mathrm{d}^{2} \delta_{1}}{\mathrm{dt}^{2}}+\mathrm{P}_{\mathrm{d} 1} \frac{\mathrm{d} \delta_{1}}{\mathrm{dt}}=\mathrm{P}_{10}-\mathrm{P}_{1}\left(\delta_{12}, \delta_{13}, \ldots \delta_{1 \mathrm{n}}\right) ; \\
& \mathrm{T}_{\mathrm{j} 2} \frac{\mathrm{d}^{2} \delta_{2}}{\mathrm{dt}^{2}}+\mathrm{P}_{\mathrm{d} 2} \frac{\mathrm{d} \delta_{2}}{\mathrm{dt}}=\mathrm{P}_{20}-\mathrm{P}_{2}\left(\delta_{12}, \delta_{13}, \ldots \delta_{1 \mathrm{n}}\right) ; \\
& \mathrm{T}_{\mathrm{jn}} \frac{\mathrm{d}^{2} \delta_{\mathrm{n}}}{\mathrm{dt}^{2}}+\mathrm{P}_{\mathrm{dn}} \frac{\mathrm{d} \delta_{\mathrm{n}}}{\mathrm{dt}}=\mathrm{P}_{\mathrm{n} 0}-\mathrm{P}_{\mathrm{n}}\left(\delta_{12}, \delta_{13}, \ldots \delta_{1 \mathrm{n}}\right) ;
\end{aligned}
$$

- уравнения мощностей синхронных генераторов, выраженные через собственные и взаимные проводимости ветвей схемы замещения:

$$
\begin{aligned}
& \mathrm{P}_{1}=\mathrm{E}_{1}^{2} \mathrm{y}_{11} \sin \alpha_{11}+\mathrm{E}_{1} \mathrm{E}_{2} \mathrm{y}_{12} \sin \left(\delta_{12}-\alpha_{12}\right)+\ldots+\mathrm{E}_{1} \mathrm{E}_{\mathrm{n}} \mathrm{y}_{1 \mathrm{n}} \sin \left(\delta_{1 \mathrm{n}}-\alpha_{1 \mathrm{n}}\right), \\
& \mathrm{P}_{2}=\mathrm{E}_{2}^{2} \mathrm{y}_{22} \sin \alpha_{22}+\mathrm{E}_{1} \mathrm{E}_{2} \mathrm{y}_{12} \sin \left(\delta_{12}-\alpha_{12}\right)+\ldots+\mathrm{E}_{2} \mathrm{E}_{\mathrm{n}} \mathrm{y}_{2 \mathrm{n}} \sin \left(\delta_{2 \mathrm{n}}-\alpha_{2 \mathrm{n}}\right), \\
& \mathrm{P}_{\mathrm{n}}=\mathrm{E}^{2}{ }_{\mathrm{n}} \mathrm{y}_{\mathrm{nn}} \sin \alpha_{\mathrm{nn}}+\sum_{\mathrm{i} \neq \mathrm{j}}^{\mathrm{n}} \mathrm{E}_{\mathrm{i}} \mathrm{E}_{\mathrm{j}} \mathrm{y}_{\mathrm{ij}} \sin \left(\delta_{\mathrm{ij}}-\alpha_{\mathrm{ij}}\right),
\end{aligned}
$$

где $\delta_{\mathrm{i}}$ и $\delta_{\mathrm{ij}}-$ абсолютные и относительные углы нагрузки генераторов; $\mathrm{E}_{\mathrm{i}}-$ электродвижущие силы генераторов; $\mathrm{T}_{\mathrm{j}}$ - постоянные инерции агрегатов; $\mathrm{P}_{\mathrm{di}}$ - эквивалентные демпферные ко- 
эффициенты генераторов; $\mathrm{P}_{\mathrm{i}}$ - электромагнитные мощности синхронных генераторов; $\mathrm{y}_{\mathrm{ii}}, \mathrm{y}_{\mathrm{ij}}-$ собственные и взаимные проводимости системы; $\alpha_{\mathrm{ii}}$ и $\alpha_{\mathrm{ij}}$ - соответствующие дополняющие углы $[3,11]$.

Ниже приведены исходные данные и параметры сложной электрической системы (рис. 1). Параметры узлов:

G1: $\mathrm{P}_{\mathrm{Gl}}=300$ MBT; $\cos \varphi_{\mathrm{G} 1}=0.8 ; \mathrm{U}_{\mathrm{G} 1}=500 \kappa \mathrm{\kappa} ; \mathrm{T}_{\mathrm{j} 1}=6 \mathrm{c} . ; \mathrm{x}_{\mathrm{d} 1}=1.907 ; \mathrm{x}_{\mathrm{d} 1}^{\prime}=0.278$.

G2: $\mathrm{P}_{\mathrm{G} 2}=200$ MBT; $\cos \varphi_{\mathrm{G} 2}=0.8 ; \mathrm{U}_{\mathrm{G} 2}=500 \kappa \mathrm{\kappa} ; \mathrm{T}_{\mathrm{j} 2}=5.4 \mathrm{c} . ; \mathrm{x}_{\mathrm{d} 2}=1.915 ; \mathrm{x}_{\mathrm{d} 2}^{\prime}=0.275$.

Узлы соединены между собой соответствующими воздушными линиями питания L1-L4.

L1: $\mathrm{U}_{\mathrm{Ll}}=500 \kappa B ; \ell_{\mathrm{L1}}=195$ км; $\mathrm{r}_{0}=0.0397$ Ом $/$ км; $\mathrm{x}_{0}=0.31$ Ом/км.

L2: $\mathrm{U}_{\mathrm{L} 2}=500 \mathrm{\kappa B} ; \ell_{\mathrm{L} 2}=115$ км; $\mathrm{r}_{0}=0.0362$ Ом/км; $\mathrm{x}_{0}=0.306$ Ом/км.

L3: $\mathrm{U}_{\mathrm{L} 3}=500 \mathrm{\kappa B} ; \ell_{\mathrm{L} 3}=180$ км; $\mathrm{r}_{0}=0.0397$ Ом/км; $\mathrm{x}_{0}=0.31$ Ом/км.

L4: $\mathrm{U}_{\mathrm{L} 4}=500$ кB; $\ell_{\mathrm{L} 4}=175$ км; $\mathrm{r}_{0}=0.0397$ Ом/км; $\mathrm{x}_{0}=0.31$ Ом/км.

Параметры нагрузочного узла:

$\mathrm{P}_{\text {Load }}=600$ МВт; $\cos \varphi_{\text {Load }}=0.88 ; \mathrm{U}_{\text {Load }}=500$ кB.

Генераторы оснащены автоматическими регуляторами возбуждения сильного действия, реагирующими на отклонение и первую производную угла, а также на отклонение напряжения. Выполним расчет установившегося режима и проверим положительность первого минора $\mathrm{q}_{11}$ матрицы квадратичной формы Q для генерирующих узлов.

Результаты расчета установившегося режима:

$$
\dot{\mathrm{U}}_{1}=505,2 \mathrm{e}^{\mathrm{j} 15^{\circ}} \kappa \mathrm{B}, \dot{\mathrm{U}}_{2}=496,2 \mathrm{e}^{\mathrm{j} 34^{\circ}} \kappa \mathrm{B}, \dot{\mathrm{U}}_{3}=509,8 \mathrm{e}^{\mathrm{j} 23^{\circ}} \kappa \mathrm{B} .
$$

Затем, используя полученные данные, решим уравнение функции Ляпунова в квадратичной форме относительно указанных выше систем уравнений (5).

На рис. 2 отражено изменение первого диагонального минимума $\mathrm{q}_{11}$ матрицы Q функции Ляпунова в квадратичной форме, показывающий предел передачи по статической устойчивости.

Расчет производили с утяжелением режима - постепенным увеличением нагрузки $\mathrm{P}_{\text {Load }}=150$ MВт до $\mathrm{P}_{\text {Load } \max }=200$ МВт, что приводит к увеличению угла до $\delta_{\text {cr }}=138^{\circ}$.

\section{Выводы}

На основе исследований были получены два важных результата.

1. Устойчивость электрической системы при малых отклонениях характеризуется положительностью первого минора матрицы квадратичной формы q $11>0$ функции Ляпунова в квадратичной форме. Этот критерий называется упрощенным [3, 11], так как его положительность определяет положительность высших миноров матрицы. Важно, что условие $\mathrm{q}_{11}>0$ содержит теоретически известные типы нарушения устойчивости электрической системы и, следовательно, как необходимые, так и достаточные условия устойчивости.

$$
-936-
$$




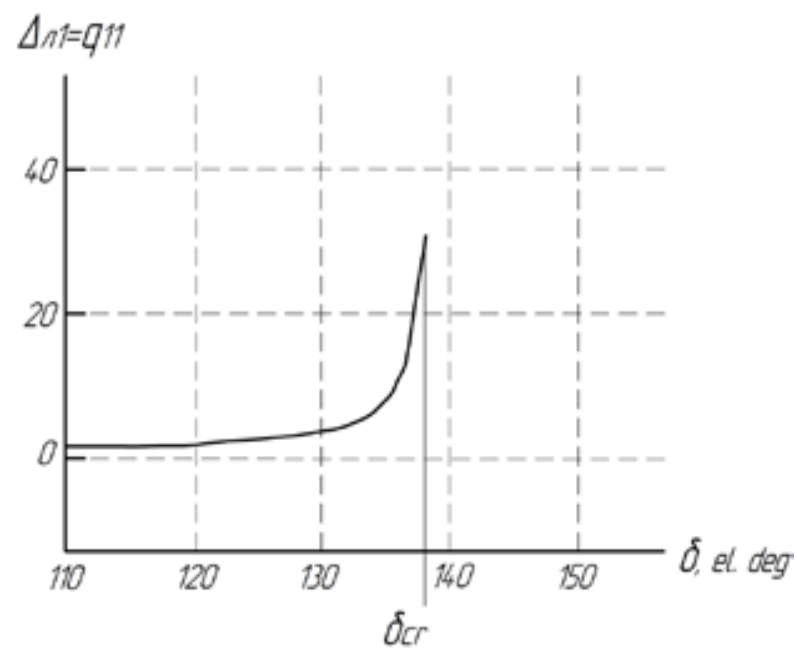

Рис. 2. Характер изменения первого диагонального минора $\mathrm{q}_{11}$ матрицы Q функции Ляпунова в квадратичной форме

Fig. 2. The character of the change of the first diagonal minor $\mathrm{q}_{11}$ of the matrix $\mathrm{Q}$ of the Lyapunov`s function in quadratic form

2. Аналитически определяется і-й генератор сложной электрической системы, первым приближающийся к пределу устойчивости:

$$
\frac{\mathrm{dq}_{11 \mathrm{j}}}{\mathrm{d} \Pi} \rightarrow \max ,
$$

где П - какой-либо параметр.

Отличительной особенностью этого подхода было разрешение уравнений состояния системы относительно абсолютных углов в отличие от традиционных уравнений системы, составленных по взаимным углам [3, 11]. Этот подход упрощает изучение переходных режимов электрических систем, в том числе при анализе их статической устойчивости.

По мнению авторов, исследования малых колебаний электрической системы на основе функций Ляпунова в квадратичной форме должны быть разработаны и проведены по направлениям:

- усовершенствование модели уравнений узловых напряжений для совместного применения с функцией Ляпунова в квадратичной форме;

- разработка более точной модели электрической системы;

- разработка матричных методов для совокупного и взаимосвязанного оптимального управления между подстанциями и станциями электрической системы;

- разработка алгоритма и модели для оптимального управления, оценки и синтеза соответствующих законов управления ЭЭС при вероятностном характере исходной информации.

\section{Список литературы}

[1] Anderson P.M., Fouad A.A. Power system control and stability, Second edition, Willeyinterscience, USA, 2003, $672 \mathrm{p}$. 
[2] Albertos A.P., Sala A. Multivariable control systems, Springer, 2007, 340 p.

[3] Аллаев К.Р., Мирзабаев А.М. Малые колебания электрических систем. Ташкент: Fan va tehnologiya, 2011. 325 c. [Allaev K.R., Mirzabaev A.M. Small oscillations of electrical systems. Tashkent, Fan va tehnologiya, 2011, 325 p. (in Russian)]

[4] Abdellatif Ben Makhlouf. Stability with respect to part of the variables of nonlinear Caputo fractional differential equations. Mathematical Communications, 2018, 23, 119-126.

[5] Kunder P. Power system stability and control, McGraw-Hill, Inc., USA, 1993, 1196 p.

[6] Debeljkovic D.Lj., Buzurovic I.M. and Simeunovic G.V. Stability of linear discrete descriptor systems in the sense of Lyapunov. International journal of information and systems sciences, 2011, 7(4), 303-322.

[7] Фазылов Х.Ф., Насыров Т.Х. Установившиеся режимы электроэнергетических систем и их оптимизация. Ташкент: Молия, 1999. 370 с. [Fazylov Kh.F., Nasyrov T.H. Established regimes of electric power systems and their optimization, Tashkent, Moliya, 1999, 370 p. (in Russian)]

[8] Allaev K.R., Mirzabaev A.M., Makhmudov T.F., Makhkamov T.A. Matrix Analysis of Steady-State Stability of Electric Power Systems. AASCIT Communications, 2015, 2(3), 74-81.

[9] Буков В.Н. Вложение систем. Аналитический подход к анализу и синтезу матричhblx систем. Калуга: Издательство Н.Ф. Бочкаревой, 2006. 720 с. [Bukov V.N. Embedding systems. Analytical approach to the analysis and synthesis of matrix systems. Kaluga, Publisher N.F. Bochkareva, 2006, 720 p. (in Russian)]

[10]Мисриханов М.Ш. Классические и новые методы анализа многомерных динамических систем. М.: Энергоатомиздат, 2004. 566 c. [Misrikhanov M.Sh. Classical and new methods for the analysis of multidimensional dynamic systems, Moscow, Energoatomizdat, 2004, 566 p. (in Russian)]

[11] Аллаев К.Р., Мирзабаев А.М. Матричные методы анализа малых колебаний электрических систем. Ташкент: Fan va tehnologiya, 2016. 432 с. [Allaev K.R., Mirzabaev A.M. Matrix methods for the analysis of small oscillations in electrical systems, Tashkent, Fan va tehnologiya, 2016, 432 p. (in Russian)] 